\title{
Projets douleur en médecine vétérinaire
}

\section{Pain Projects in Veterinary Medicine}

\author{
T. Poitte \\ (C) Lavoisier SAS 2019
}

\begin{abstract}
Résumé Dans le monde vivant, nous partageons la douleur avec les animaux. Dans le monde des professionnels de santé, la prise en charge de la douleur est un serment ( je ferai tout pour soulager les souffrances ») ou une obligation déontologique (" je m'efforce d'atténuer la souffrance de l'animal ») exposant la douleur comme le cœur de métier des médecins et des vétérinaires. Longtemps des freins d'origines diverses (dolorisme médical, spécisme, pauvreté de l'arsenal thérapeutique...) ont entravé ces règles et cette aspiration. Aujourd'hui, en médecine vétérinaire, des progrès considérables ont été réalisés, mais les données actuelles des neurosciences et de l'éthologie nous encouragent à transformer ce cœur de métier en cœur de compétences : centrale et vitale, la douleur devient le liant entre les compétences individuelles, mais souvent isolées des vétérinaires. Immanente mais interdisciplinaire, la douleur fait plus qu'additionner des spécialités en créant une synergie de compétences au sein d'un projet douleur de toute l'équipe d'un établissement de soins vétérinaires. Cet article traite des projets douleur en médecine vétérinaire, cherchant à répondre à cette ambition et à la préoccupation sociétale du bien-être animal.
\end{abstract}

Mots clés Douleur animale · Bien-être animal · e-santé · Réseau douleur $\cdot$ Consultation douleur

\begin{abstract}
In the world of living beings, both humans and animals experience pain. In the world of health professionals, pain management is a pledge ( $I$ shall do everything to alleviate suffering) or an ethical obligation (I strive to relieve animal suffering), revealing pain as the core professional concern for doctors and veterinarians. For a long time, various constraints (medical sanctification of suffering, speciesism, the inadequacy of the therapeutic arsenal,
\end{abstract}

\section{T. Poitte $(\square)$}

Clinique vétérinaire La croix Michaud

F-17630 La Flotte en Ré, France

e-mail : thierry.poitte@capdouleur.fr etc.) have hindered these rules and this aspiration. These days considerable progress has been made in veterinary medicine, but recent data from neuroscience and ethology spur us on to turn this core professional concern into core skills: central and critical, pain becomes the bond between veterinarians' separate and often isolated skills. Inherent but interdisciplinary, pain does more than bring specialisms together; it creates synergy between skills in a pain project for a veterinary care institution's entire team. This article looks at pain projects in veterinary medicine, hoping to respond to this ambition and society's concerns around animal welfare.

Keywords Animal pain - Animal Wellbeing - e-health · Pain Network · Pain Consultation

La douleur de l'enfant et la douleur animale ont une histoire en miroir tragique : celle d'avoir été longtemps niée, pour cause d'immaturité du système nerveux pour les premiers, en raison, pour les seconds, d'une absence cartésienne d'âme, de langage et donc... de raison. Les explications du déni de la douleur néonatale ont été identifiées : hyposensibilité des enfants nouveau-nés, immaturité du système nerveux, absence de souvenir de la douleur dans les premières années [1]. Ce sont aussi les travaux de Anand et Hickey [2] sur les bénéfices des morphiniques au cours de la chirurgie du canal artériel chez les bébés prématurés qui révolutionnèrent définitivement cette hyposensibilité légendaire : le concept neuroanatomique des voies de la douleur est bien applicable aux prématurés. Pire encore : l'inadéquation entre les voies ascendantes excitatrices et les voies descendantes inhibitrices fragilise davantage le nouveau-né qui garde des traces mnésiques de douleur dans un cortex à la plasticité exacerbée [3].

Concernant la douleur animale, parlons plutôt de révolution lente : les progrès des neurosciences comparées, les apports de l'éthologie et de la psychologie animale ont encore à ferrailler aujourd'hui contre l'ancrage culturel de croyances religieuses, d'anciennes pensées philosophiques 
et des récentes positions créationnistes ou spécistes, légitimant la supériorité de la condition humaine.

Contre toute éthique et légitimité scientifique, le dolorisme médical et ses pseudovertus de diagnostic ou de garde-fou naturel persistent encore aujourd'hui.

Actuellement, la douleur animale se confronte, à des degrés divers, à quatre étapes successives de méconnaissances :

- de l'existence même de la douleur chez toutes les espèces et de la compréhension de ses mécanismes : «Je ne crois pas que la douleur existe chez tous les animaux»;

- de l'importance des conséquences physiopathologiques des processus douloureux et des états de vulnérabilité de l'animal souffrant : "Je sais que la douleur animale existe, mais je ne suis pas convaincu de ses effets délétères; je pense que la douleur animale est bien supportée et qu'elle a des vertus diagnostiques et/ou thérapeutiques »;

- de l'ignorance des solutions thérapeutiques innovantes ou des référentiels actualisés : «Je connais la réalité de la douleur animale et de ses impacts négatifs sur la santé et le bien-être animal : malheureusement, j'ignore les moyens pharmacologiques et les méthodes non pharmacologiques »;

- des difficultés du praticien à mettre en œuvre ces nouvelles solutions thérapeutiques : «J'accepte bien volon- tiers les recommandations de prise en charge de la douleur animale, mais je ne me sens pas capable de les appliquer dans ma pratique quotidienne ».

Pour améliorer la prise en charge de la douleur animale, les solutions uniques sont inopérantes contre ces blocages successifs : inutile d'apporter des réponses à l'incapacité de mise en œuvre si les niveaux inférieurs de méconnaissances ne sont pas traités. Chaque croyance négative doit donc être identifiée puis combattue par un argumentaire solide, propre à réveiller la conscience scientifique du praticien, ses compétences en éthologie pour une meilleure évaluation de la douleur, ses connaissances en pharmacologie, son adaptation aux méthodes complémentaires, enfin sa capacité à lever les freins au changement et à manager une équipe soignante autour d'un projet éthique.

Les nombreux projets douleur répondent actuellement à ces difficultés grâce aux innovations thérapeutiques proposées par les laboratoires, à la m-santé pour les pratiques évaluatives, à la télémédecine pour le suivi, à la formation continue et à ses nouveaux formats d'apprentissage, à la mise en réseau des vétérinaires pour améliorer la connaissance des douleurs et la pertinence des solutions thérapeutiques, à la création des unités vétérinaires d'évaluation et de traitement de la douleur (UVETD) et enfin à l'engagement dans l'amélioration de la qualité de vie (bien-être animal) (Fig. 1).

\section{E-santé \& Douleur}

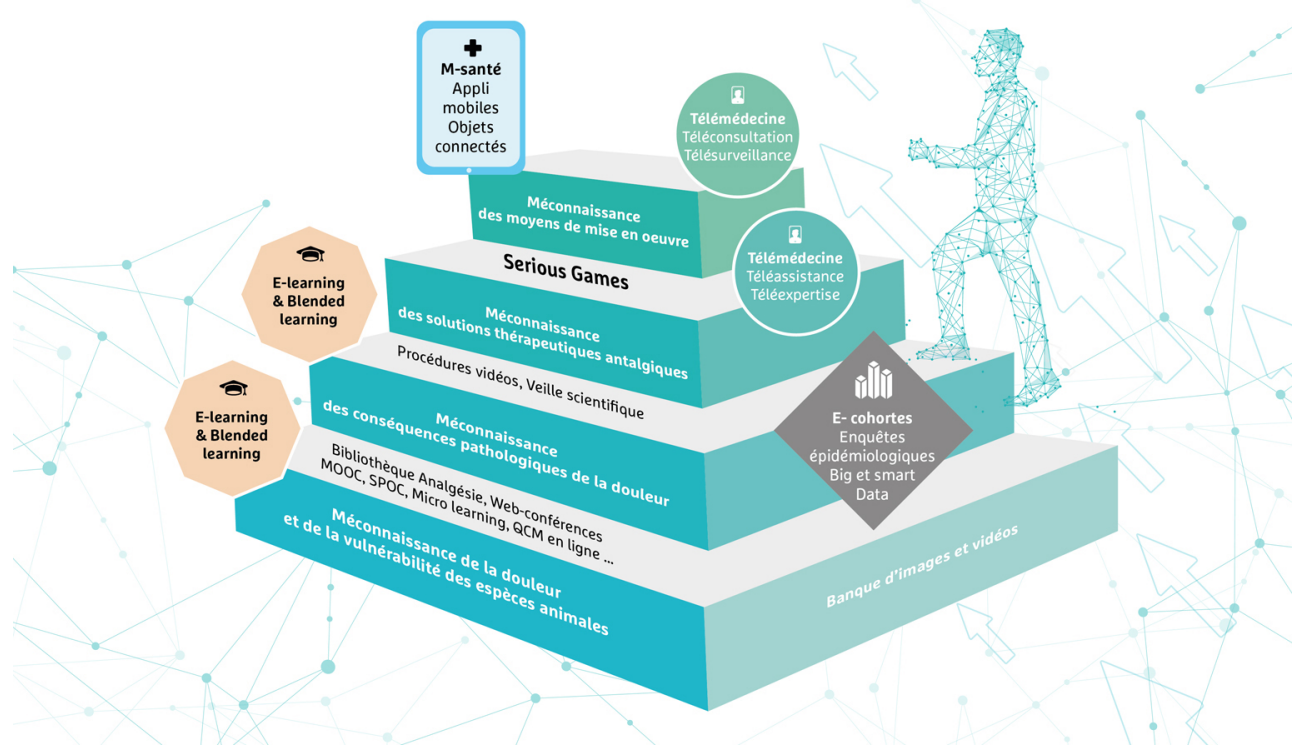

Fig. 1 Correction des quatre niveaux de méconnaissances de la douleur et de sa prise en charge par l'approche de la e-santé. Abréviations : Mooc : massive open online course ; QCM : questionnaire à choix multiples ; Spoc : small private online course. D'après Morgan Cahouet Réseau CAPdouleur 


\section{Innovations thérapeutiques en médecine vétérinaire}

\section{Anti-inflammatoires}

La très forte sélectivité COX-2 (cyclo-oxygénases de type 2) des nouveaux anti-inflammatoires non stéroïdiens (AINS, comme le firocoxib, le cimicoxib et le robenacoxib) n'améliore pas significativement la tolérance aux effets digestifs indésirables par rapport aux préférentiels COX-2. Leur intérêt réside dans la thérapie adjuvante anticancéreuse pour les tumeurs surexprimant les COX-2 (seuls ou en association avec un protocole de chimiothérapie ou de radiothérapie) : carcinomes nasaux, mammaires, transitionnels de la vessie, épidermoïdes... [4,5].

Le grapiprant de la famille des piprants est un AINS non inhibiteur des COX mais antagoniste compétitif des récepteurs EP4 des PGE2 pro-inflammatoires [6]. L'affinité nulle pour les récepteurs EP1, EP2 et EP3 évite le blocage des prostaglandines constitutives (sécrétion du mucus gastroprotecteur et filtration rénale). Très séduisant sur le plan théorique, le grapiprant n'est recommandé, selon le résumé des caractéristiques du produit (RCP), que pour le traitement de la douleur associée à une arthrose peu sévère à modérée chez le chien. Il entraîne très fréquemment des vomissements, non associés à des érosions ou ulcérations digestives.

\section{Antalgiques à base d'opiö̈des}

Le tramadol a été récemment approuvé en France dans le cadre d'une procédure d'autorisation d'un médicament générique vétérinaire. Le comité de suivi de l'Agence nationale du médicament vétérinaire (ANMV) a rédigé un avis soulignant l'efficacité limitée du tramadol chez toutes les races de chiens en raison d'une absorption par voie orale très faible (et très variable) ainsi qu'une conversion minime en métabolite actif sur les récepteurs opioïdes des chiens, y compris après une injection. L'étude clinique [7] citée par l'ANMV est toutefois contestable par la présence d'un certain nombre de biais, dont la sélection de formats de chiens très différents $(5$ à $50 \mathrm{~kg})$, de différents types d'arthrose (coude et grasset), des méthodes d'évaluation (plateau de forces et Canine Brief Pain Inventory) s'intéressant plus à la fonction qu'au ressenti émotionnel et donc aux répercussions comportementales des douleurs chroniques. Les observations cliniques et une enquête sur les pratiques de prescription ont démontré le décalage entre le manque de preuves de la médecine factuelle (essai clinique randomisé croisé en double insu contre placebo et substance active) et l'utilisation plébiscitée par une large majorité de cliniciens (dû probablement à l'action inhibitrice de la recapture de la noradrénaline et de la sérotonine).

\section{Antiépileptiques et antidépresseurs}

La gabapentine est de plus en plus prescrite en médecine vétérinaire à la dose de 5 à $10 \mathrm{mg} / \mathrm{kg}$, deux ou trois fois par jour, pour la prise en charge des douleurs neuropathiques [8]. Le dosage est individuel, et il est à adapter en augmentant progressivement la dose pour éviter une sédation excessive. Il est d'usage de stabiliser l'animal (plusieurs semaines) avant d'envisager de le sevrer en diminuant les doses (paliers de quart de dose). Les effets secondaires digestifs (vomissements et diarrhée) sont rares, et il n'y a pas de contre-indication à son emploi. En cas d'inefficacité, la gabapentine peut être associée aux antidépresseurs tricycliques (clomipramine).

\section{Cannabinoïdes}

Comme en médecine humaine, le cannabis suscite un fort intérêt en relation avec ses bénéfices sur le ressenti émotionnel de la douleur des chiens et chats : il y a cependant un risque de banalisation à cause du développement par des laboratoires de compléments alimentaires à destination du confort des animaux âgés. Dans l'ensemble des études de qualité portant sur les douleurs chroniques chez l'Homme [9], les cannabinoïdes ont eu un effet analgésique modéré mais souvent associé à des effets secondaires (dysphorie, confusion, vertige, tachycardie dose-dépendante). Le cannabidiol pourrait être intéressant dans la prise en charge des douleurs chroniques chez les animaux de compagnie en raison d'effets anxiolytiques, antidépresseurs et d'effets bénéfiques sur la qualité du sommeil, venant ainsi diminuer la perception de la douleur et améliorer la qualité de vie.

Le cannabis thérapeutique devrait être exclusivement utilisé pour le traitement des douleurs réfractaires chroniques notamment neuropathiques (syringomyélie, hernies discales, arthrose, douleurs postopératoires, douleurs cancéreuses, syndrome d'hyperesthésie féline...) et pour l'accompagnement des animaux douloureux en fin de vie.

La procédure à suivre est du type start low, go slow et stay low, c'est-à-dire de pratiquer une titration progressive et précautionneuse, avec un étalement des doses de 0,1 à $4 \mathrm{mg} / \mathrm{kg}$ chez le chien, couplée à une évaluation rigoureuse de la douleur (par exemple avec le système de gradation ClientSpecific Outcome Measures, CSOM), au sein d'une relation privilégiée avec le propriétaire, en accord avec des objectifs partagés d'amélioration de la qualité de vie. La profession vétérinaire a besoin de progresser sur la pertinence des indications, la compréhension des interactions médicamenteuses (avec les inducteurs enzymatiques et les inhibiteurs des cytochromes P450), la dangerosité des effets indésirables. Au Québec, où l'usage médical du cannabis est légal chez l'Homme (interdit chez le chien), c'est la troisième cause d'intoxication chez les chiens. Comme pour son utilisation 
chez l'Homme, la prescription devra être particulièrement encadrée (recours à la préparation magistrale, ordonnance sécurisée, registres...) dans un double objectif de sécurité et d'analyses des effets bénéfiques.

\section{Biothérapies}

Le plasma riche en plaquettes (PRP) est un produit sanguin autologue, dont les effets bénéfiques (accélération de la cicatrisation, diminution de l'inflammation) reposent sur des facteurs de croissance présents dans le plasma et libérés par les plaquettes [10]. Du plasma autologue est conditionné en moins de cinq minutes à partir d'un prélèvement de sang total. La préparation stérile obtenue par centrifugation permet l'injection intra-articulaire sur le site arthrosique, sous contrôle échographique si nécessaire. Il n'existe pas de consensus à propos du protocole à suivre, mais 60 à $75 \%$ de bons à très bons résultats ont été observés sur l'expression douloureuse associée (cas cliniques évalués à l'aide de Dolodog et colliers d'activités) avec deux injections à un mois d'intervalle, suivies d'un rappel six à huit mois après. Le recours au PRP contre-indique l'emploi des AINS dans le même temps. Outre l'arthrose, les indications du PRP incluent les tendinites et la gestion des plaies complexes.

Les cellules souches mésenchymateuses (CSM) sont utilisées dans le cadre de la médecine régénérative vétérinaire pour leurs multiples vertus : capacités de différenciation en cellules spécialisées (ostéoblastes, chondroblastes, fibroblastes, adipocytes), fonctions trophiques chondroprotectrices et fonctions immunomodulatrices. Les articulations sont traitées par injection intra-articulaire unique sous sédation ou anesthésie générale. Plusieurs études récentes ont confirmé l'innocuité et l'efficacité à long terme des CSM dans le traitement de l'arthrose canine [11,12].

Les anticorps monoclonaux, produits en laboratoire à partir d'un seul clone de lymphocytes $\mathrm{B}$ sont déjà utilisés en médecine vétérinaire pour le traitement de l'atopie canine. En analgésie, la cible spécifique est le nerve growth factor (NGF), neurotrophine indispensable au développement et à la différenciation du système sympathique et des neurones sensoriels chez l'embryon.

Chez l'adulte, le NGF est un médiateur important de l'inflammation et plus spécifiquement de la douleur en amplifiant la réponse des canaux TRPV1, ASIC, P2X et les récepteurs à la bradykinine, en augmentant la libération de substance $\mathrm{P}$ et de CGRP, en promouvant le couplage entre les afférences sympathiques et les nocicepteurs et en activant les récepteurs AMPA, NMDA et NK1 de la corne dorsale. Le NGF est sécrété en grande quantité dans les articulations enflammées, et il est considéré comme l'un des principaux acteurs de l'hyperalgésie primaire induite par l'inflammation périphérique. En médecine vétérinaire, le NV-01 (anti-NGF recombinant canin) a été utilisé ( $200 \mu \mathrm{g} / \mathrm{kg}$ i.v.) pour la prise en charge de la douleur associée à l'arthrose au cours d'une étude portant sur 26 chiens : l'amélioration clinique a été objectivée par l'évaluation des scores de douleur (plateau de forces et grille CBPI, Canine Brief Pain Inventory) et perdure au moins quatre semaines [13]. Le NV-02 (antiNGF recombinant félin) a augmenté significativement pendant six semaines les mesures d'activité, les scores des Client-Specific Outcome Measures et de la grille FMPI (Feline Musculoskeletal Pain Index) chez 34 chats arthrosiques [14].

Les anticorps monoclonaux présentent des caractéristiques pharmacologiques uniques qui seront particulièrement profitables chez le chat arthrosique aux comorbidités très fréquentes (insuffisance rénale, hyperthyroïdie, diabète...) et à la prise de médicaments difficile : cible spécifique avec un mode d'action $\mathrm{Ac} / \mathrm{Ag}$, demi-vie très longue par injection, pas de métabolisation par les enzymes hépatiques ou rénales, pas d'excrétion urinaire, rares interactions médicamenteuses...

\section{M-santé et évaluation de la douleur}

Définie par l'Organisation mondiale de la santé (OMS) en 2009, la m-santé regroupe la pratique médicale ou la collecte de données sur tout dispositif mobile : smartphone, tablette, objet connecté.

\section{Suivi des douleurs arthrosiques}

L'arthrose est une maladie pluritissulaire dégradative et inflammatoire. Les douleurs arthrosiques ont pour origine le compartiment articulaire (douleurs mécaniques liées à l'exercice, douleurs inflammatoires nocturnes, douleurs neuropathiques spontanées de type « décharges électriques ») et le compartiment central (hyperalgésie et allodynie). Les douleurs deviennent chroniques, c'est-à-dire définies comme persistantes ou récurrentes, induisant une détérioration fonctionnelle et des perturbations émotionnelles venant altérer significativement le comportement et la qualité de vie de l'animal. Les douleurs arthrosiques évoluent en fonction de très nombreux paramètres : progression de la maladie, sensibilité interindividuelle, conditions environnementales (climat, mode de vie, relation propriétaire-animal), nutrition, exercice, efficacité thérapeutique... Les douleurs arthrosiques peuvent être recherchées lors de l'examen clinique du praticien et par la visualisation des changements structuraux (imagerie articulaire), sans qu'il y ait pour autant une corrélation exacte entre la sévérité des réactions ou des lésions et le ressenti douloureux de l'animal. L'évaluation de la fonction locomotrice (score de boiterie) doit être complétée par l'étude des composantes émotionnelles et comportementales de la douleur, grâce à des grilles multiparamétriques 
validées : Helsinki, Liverpool ou Canine Brief Pain Inventory [15-17].

\section{Développement d'applications évaluant la douleur}

Les pratiques évaluatives pour la prise en charge des douleurs chroniques sont très peu répandues en médecine vétérinaire. La grande majorité des praticiens n'utilise pas de grilles d'évaluation par manque de temps, de connaissances ou de reconnaissance de leur intérêt. Le réseau CAPdouleur a créé l'application web Dolodog, dérivée de la grille d'Helsinki, destinée aux cliniques vétérinaires, pour les aider à évaluer la douleur des animaux qui leur sont confiés et optimiser le suivi des prescriptions (Fig. 2). La grille d'Helsinki développée spécifiquement pour la douleur arthrosique a été modifiée pour tenir compte des signes cliniques d'hypersensibilisation (hyperalgésie et allodynie), des répercussions comportementales de la chronicisation de la douleur (irritabilité ou agressivité) et des situations évocatrices de douleurs neuropathiques (douleurs spontanées de type « décharges électriques »). La version électronique permet une évalua-

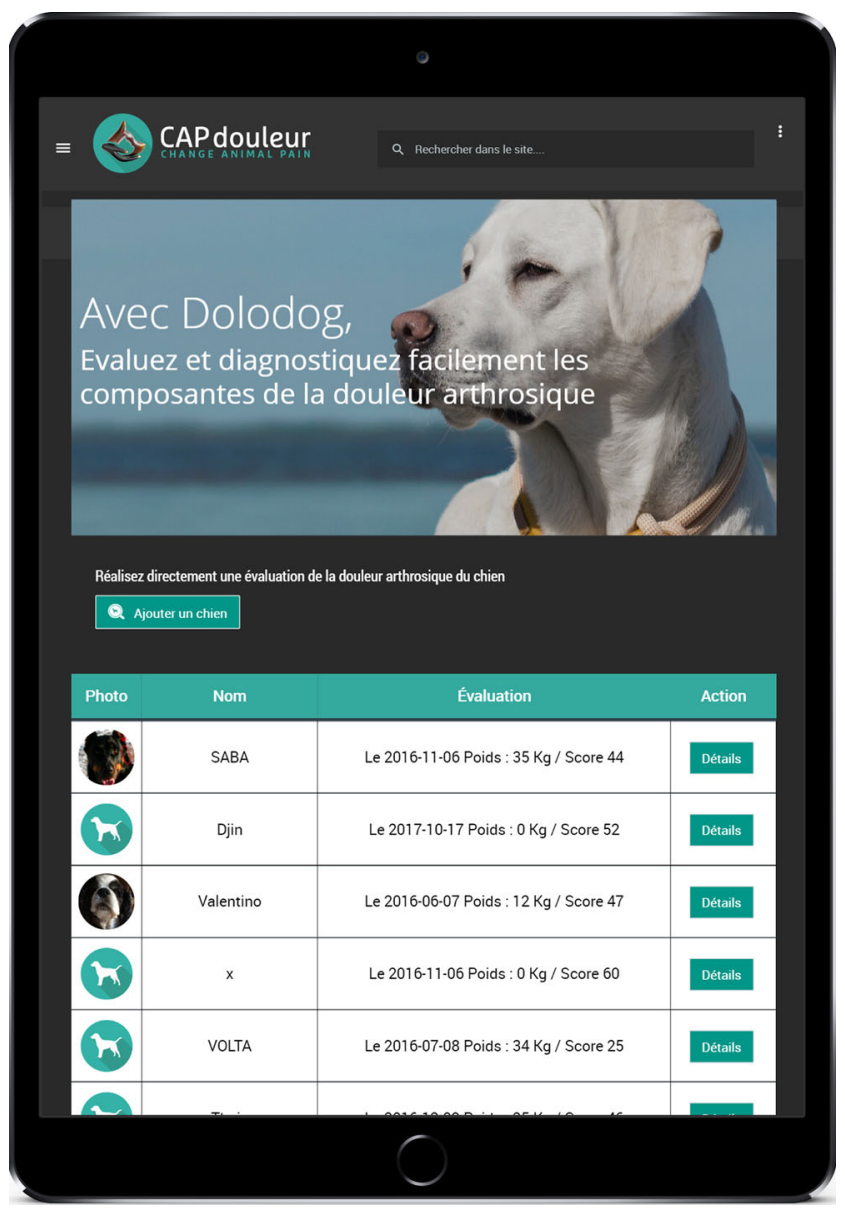

Fig. 2 Web application Dolodog permettant l'évaluation des douleurs arthrosiques tion qualitative (diagnostic des composantes neuropathiques et centrales) et quantitative de la douleur (intensité des douleurs inflammatoires). Dolodog améliore la pertinence des choix thérapeutiques : respect des paliers I, II et III pour les douleurs inflammatoires, antiépileptiques et antidépresseurs tricycliques pour les douleurs neuropathiques, antiNMDA (N-méthyl-D-aspartate) pour les douleurs centrales. Dolodog autorise le suivi des thérapeutiques pharmacologiques ou non, en facilitant l'archivage des données. Dolodog place le vétérinaire et le propriétaire dans une relation d'alliance thérapeutique, clé d'une observance améliorée pour la prise en charge des douleurs chroniques. Plus de 1800 évaluations Dolodog ont été collectées à ce jour : elles permettent déjà de mieux connaître la maladie arthrosique et les proportions relatives de chaque composante de la douleur. Les conclusions seront publiées prochainement.

\section{Intérêts des accéléromètres}

Les douleurs arthrosiques sont à l'origine d'une diminution de l'activité physique par gêne et inconfort fonctionnel, en raison des contraintes mécaniques et des nombreux stimuli nociceptifs que l'animal arthrosique perçoit. La dépression associée réduit l'envie de mobilité, tandis que les troubles du sommeil perturbent le repos nocturne en raison de l'inconfort de la position, mais aussi de l'abaissement du seuil douloureux. Les composantes fonctionnelles et émotionnelles de la douleur peuvent être mesurées objectivement par l'accélérométrie. Des études vétérinaires récentes ont validé l'accélérométrie comme outil d'évaluation de l'activité physique et de réussite d'un traitement anti-inflammatoire chez des chiens arthrosiques $[18,19]$. Positionné à proximité du centre de gravité, l'accéléromètre permet de mesurer l'activité motrice du chien, en faisant la différence entre la marche, la course, le jeu et le sommeil. L'analyse des accélérations permet de déterminer l'asymétrie et la régularité de la démarche, donc d'affiner le degré de boiterie présente ou encore de diagnostiquer des boiteries indétectables à l'œil nu. L'actimétrie a l'avantage, sur les tapis de marche, de permettre le suivi continu des chiens dans leur environnement quotidien.

Les objets connectés proposés actuellement en France n'offrent pas suffisamment de services et de garanties pour évaluer les douleurs chroniques : manque d'autonomie, taille et poids des dispositifs, pertinence des algorithmes, synchronisation des données, absence d'interface dédiée aux professionnels de santé, problème d'intégration aux logiciels métier, etc. La valeur d'usage n'a pas été suffisamment travaillée en amont avec les vétérinaires, ce qui explique aussi le principal frein à l'équipement, par manque d'utilité ressentie des objets connectés. L'objet connecté doit s'inscrire dans une prise en charge globale de la douleur chronique, pariant sur la complémentarité des données objectives brutes et mathématiques, avec une approche subjective plus subtile, 
humaine et empathique, compréhensive des mécanismes complexes et intimes de la douleur. Une évaluation maîtrisée de la douleur reliant l'indispensable relation humaine avec le propriétaire (médecine narrative) aux outils innovants (Dolodog et tracker d'activité) est la première étape d'une alliance thérapeutique fondée sur un climat de compétences et de confiance. L'objet connecté sera d'autant plus utile pour les praticiens que les données récoltées seront partagées avec l'ensemble de la profession vétérinaire via des réseaux scientifiques. L'utilité de l'objet connecté doit aussi être démontrée aux propriétaires. Pour cela, l'objet doit avoir été conçu en fonction des besoins, des attentes et des contraintes voulues et/ou subies par les utilisateurs : praticité, autonomie, garantie...

Une double stratégie de motivation dirigée vers le praticien et le propriétaire peut, par exemple, s'appuyer sur l'offre différenciante de la consultation douleur.

La «gamification » est l'utilisation des mécanismes du jeu pour faciliter l'apprentissage. Se fixer des défis par le jeu est l'une des caractéristiques du numérique qui suscite le plus d'attraction chez les utilisateurs pour acheter, puis pour utiliser les accessoires connectés (IoT pour Internet of things). Le défi peut être individuel (amélioration de la mobilité de son animal après traitement) ou collectif (comparaison de la mobilité de son animal souffrant à une population de chiens de même race et atteints de la même maladie chronique). Certains moniteurs convertissent l'activité (marche, course, jeu) et la durée du sommeil en points : un objectif de points, reflet de données statistiques globalisées, et censé représenter le bien-être de l'animal est affiché sur l'écran du smartphone. Au propriétaire de relever le défi d'une meilleure qualité de vie pour son animal !

\section{Douleur et télémédecine}

Encadrée pour la première fois par le décret du 19 octobre 2010, la télémédecine est une pratique médicale à distance reposant sur cinq actes : la téléconsultation, la téléexpertise, la télésurveillance, la téléassistance et la régulation médicale. La régulation médicale est déjà une réalité dans notre profession pour les services d'urgence : VetoAdom-Alvetis traite ainsi plus de 490000 appels par an (communication personnelle de Jean-Louis Patin). Comme en médecine humaine et loin devant les traumatismes et les plaies, la douleur est le premier motif de recours aux services d'urgence. Les structures vétérinaires spécialisées dans la douleur sont rares et non reconnues pour l'instant par l'Ordre national des vétérinaires. Les consultations « douleur chronique » pour les animaux de compagnie sont insuffisamment proposées en France. La téléassistance médicale permet à un vétérinaire spécialiste d'aider ses confrères à mener ce type de consultation dédiée à la douleur et de répondre ainsi à une forte demande sociétale du respect du bien-être animal. La téléex- pertise pour les sujets de la douleur est proposée aux membres du réseau CAP douleur via une foire aux questions (FAQ), avec l'assurance d'une réponse personnalisée sous 48 heures. La solution LinkyVet développée par la société du même nom est disponible et facilite les échanges avec les experts, notamment par le partage du dossier médical multimédia de l'animal malade, sous forme de textes (examens de biologie), d'images fixes (radiographies), d'images animées (échographies) ou de sons (auscultation, plaintes, etc.). Des logiciels d'aide à la prescription d'antalgiques devraient voir le jour en 2020. La téléconsultation, dans le domaine de la douleur, ne doit s'envisager qu'après un examen clinique préalable : le contact physique avec l'animal est indispensable à l'exploration de la sensibilité, de sa composante spatiotemporelle et de ses troubles (hyperalgésie, hyperpathie et allodynie). LinkyVet permet de réaliser une « visioconsultation », capable d'étoffer particulièrement la pertinence de l'évaluation de la douleur. En effet, cette dernière s'exprime différemment selon l'ambiance protectrice de la maison ou de l'environnement contraint, voire perturbant, de l'établissement de soins. Certains comportements douloureux sont associés à la prise alimentaire, à la période postprandiale, à des activités difficilement reproductibles à la clinique ou surviennent plus fréquemment la nuit ou de façon imprévisible. La visioconsultation permet le clavardage ou chat (conversation en temps réel par écran interposé) et le partage aisé de photos, de vidéos enregistrées ou de séquences en direct, dirigé par le praticien. C'est aussi un outil d'observance remarquable, qui permet aujourd'hui d'ajuster précisément la thérapeutique antalgique en tenant compte des variations individuelles et permettra aussi prochainement de relever des données issues des objets connectés. L'éducation thérapeutique initiée dans la clinique vétérinaire et prolongée à domicile gagnera en efficacité (une vidéoassistance pourrait par exemple être proposée pour des techniques de massage des chiens arthrosiques). Par rapport à des solutions existantes, LinkyVet est un outil métier facilitant les échanges et les partages avec les clients sous l'autorité du vétérinaire, qui garde la maîtrise des données, le choix du temps imparti pour la consultation et la possible monétisation de son acte à distance.

Face aux enjeux de la e-santé animale, la profession vétérinaire se doit de récolter, d'interpréter et de partager les données avec tous les praticiens pour une meilleure connaissance des maladies et des traitements, de conforter le rôle de soignant des vétérinaires en intégrant les données numériques dans le nouveau paradigme de l'alliance thérapeutique, de se rendre indispensable en donnant des attributs médicaux à la valeur d'usage des objets connectés. La création d'un label ou d'une accréditation des objets connectés par nos institutions ou des réseaux scientifiques aurait le mérite de garantir la fiabilité et la sécurité des dispositifs, la conformité avec la réglementation, la transparence sur la valeur d'usage 
et le traitement des données. L'objectif de répondre à ce futur label ou à cette accréditation justifie le partage rigoureux des données (actes médicaux, évaluations Dolodog, données actives provenant des capteurs) entre vétérinaires dans l'intérêt d'une prise en charge améliorée des douleurs chroniques arthrosiques. L'assemblage de ces données, informatisé, transparent, sécurisé et distribué à tous pourrait constituer demain le premier maillage territorial en analgésie vétérinaire.

\section{Nouveaux formats d'apprentissage}

Dans les cycles d'études médicales et vétérinaires, les formations sur la douleur représentent une vingtaine d'heures. Une offre de journées conférences et d'enseignements postuniversitaires (EPU) est proposée par l'organisme CAPdouleur (60 dates en 2019) et de soirées ou journées douleur proposées par l'Association française des vétérinaires pour animaux de compagnie (AFVAC) [trois dates en 2019].

Les formations CAPdouleur en présentiel sont fortement influencées par les acquis de l'algologie humaine et apportent des regards croisés interdisciplinaires sur les grands syndromes douloureux grâce à une analgésie raisonnée et protectrice : l'analgésie raisonnée repose sur les connaissances actualisées de la physiopathologie de la douleur. Les mécanismes mis en jeu et leur « signature » (origine) biologique sont recherchés. L'analgésie raisonnée se fonde aussi sur la connaissance des données pharmacocinétiques et pharmacodynamiques des analgésiques utilisés : mode, délai et durée d'action, voies d'administration, puissance et efficacité, effets secondaires et indésirables, contre-indications. L'analgésie raisonnée se double d'une valence protectrice, dictée par l'importance des mécanismes d'hypersensibilisation du système nerveux (hyperalgésie et allodynie). Ce concept d'analgésie protectrice, préfiguré par Dahl et Moiniche [20] puis Bromley [21], introduit la notion d'antihyperalgésie. L'objectif est de soustraire les influx nociceptifs des voies nerveuses afin de prévenir des cascades de changements neurobiologiques difficilement réversibles. La valence protectrice contrarie la progression de la douleur aiguë vers la chronicité.

Les actualités douleur sont particulièrement riches, et les vétérinaires sont demandeurs de parcours de formation continue avec un partage sans restriction des connaissances pour une appropriation rapide et opérationnelle dans leurs établissements de soins.

Les formations mixtes, CAPdouleur ou blended learning, proposent aux apprenants un accompagnement d'une ou de plusieurs formations en présentiel, associées à des formations en ligne, par exemple un audit sur l'acquisition des connaissances et l'appropriation des outils, suivi d'un webinaire sur mesure et correctif, dédié à ces mêmes apprenants.
En médecine humaine, ces programmes mixtes d'enseignement ont montré leur supériorité par rapport aux formations en présentiel seul ou en e-learning seul.

Dans le cadre de CAPdouleur, pendant six années, plus de 300 conférences ont été proposées aux praticiens dans les villes françaises. Dans un deuxième temps, les modalités d'apprentissage ont été regroupées au sein d'une plateforme internet d'informations et de services connectés pour la prise en charge de la douleur animale. Dans un troisième temps, l'ensemble des apprenants a été sollicité pour rejoindre un réseau communautaire, social et collaboratif, acteur de la esanté animale, ouvert aux praticiens généralistes et spécialistes désireux d'actualiser leur prise en charge de la douleur, par des moyens pharmacologiques ou non, des biothérapies (cellules souches, anticorps monoclonaux), des outils numériques, des services connectés et un parcours de formation continue accréditante.

\section{Mise en réseau des vétérinaires}

Le réseau CAPdouleur a été créé le 16 mai 2016 pour répondre aux besoins de formation continue des vétérinaires dans le domaine de l'analgésie. Il promeut l'interdisciplinarité avec les différentes spécialités vétérinaires et la transversalité avec la médecine humaine via la Société française d'étude et de traitement de la douleur et l'institut Analgesia (premier pôle européen de recherche et d'innovation contre la douleur). Le réseau est collaboratif puisque les membres s'engagent à partager leurs acquis et à être des acteurs de la communauté.

Le réseau permet ainsi un partage inédit d'expériences individuelles et collectives, constituant un patrimoine commun de connaissances enrichi par des articles scientifiques, des regards croisés, des banques d'images et de vidéos, des résultats d'enquêtes sur la prévalence d'affections chroniques douloureuses ou de pratiques de prescription. Un réseau peut aussi compléter la recherche traditionnelle trop fortement dépendante du gold standard représenté par l'essai témoin randomisé : sélection drastique des malades, nombreux critères d'exclusion éloignant cette pratique de la réalité des soignants, durée d'étude courte, barrières administratives, etc. En pratique quotidienne, ces nouveaux traitements sont utilisés de manière prolongée chez des malades porteurs de plusieurs affections, et les résultats observés peuvent alors être très différents. Via la constitution de banques de données et de cohortes épidémiologiques, le réseau propose la mise en place d'essais pragmatiques sur de larges populations : critères d'inclusion plus souples tenant compte des polypathologies des animaux âgés, sélection des animaux à traiter par analyse de bases de données, randomisation centrée sur les habitudes du vétérinaire prescripteur, suivi longitudinal de longue durée, critères d'analyse orientés vers la qualité de 
vie. Ainsi, le réseau CAPdouleur récolte les données issues de ses enquêtes sur les pratiques de prescription, de ses applications web et des prochains objets connectés validés mis à la disposition des membres du réseau. Il interprète les résultats en vue d'approfondir les connaissances autour de l'évaluation et des traitements de la douleur. L'ensemble des données est partagé avec la profession vétérinaire. À ce jour $\left(1^{\mathrm{er}}\right.$ septembre 2019), il fédère 350 cliniques, cabinets ou centres hospitaliers vétérinaires, soit 1200 praticiens vétérinaires. Certes, les données collectées ne sont pas massives en volumétrie, mais elles apparaissent significatives (15\% des praticiens canins sont adhérents au réseau) et, surtout, ciblées sur des informations qualitatives, fiables et prometteuses, au chevet des pratiques, intelligentes et donc immédiatement interprétables. Celles-ci préfigurent les enjeux réalistes d'une médecine prédictive, préventive, personnalisée, participative et pertinente.

\section{Offre de consultation douleur}

La consultation douleur est proposée aux propriétaires de chiens ou de chats présentant des affections locomotrices et/ou des troubles du comportement, des douleurs chroniques postopératoires, subissant des protocoles de chimiothérapie ou de radiothérapie ou encore d'animaux de compagnie en fin de vie.

Les objectifs sont :

- de réaliser un examen clinique complet, assorti d'analyses complémentaires si nécessaire, afin de rechercher avec précautions toute affection potentiellement douloureuse ;

- d'apprendre au propriétaire à reconnaître et à évaluer les signes de douleur, fondés sur l'observation de troubles fonctionnels et de modifications comportementales spécifiques du chien ou du chat ;

- de choisir ensemble (vétérinaire et propriétaire) les moyens médicamenteux et les thérapeutiques complémentaires ou alternatives (biothérapies, physiothérapie, acupuncture, diététique...) pour soulager durablement l'animal douloureux.

La consultation douleur offre un cadre formalisé de cinq étapes pour la prise en charge plurimodale et pluridisciplinaire des douleurs chroniques :

- anamnèse et médecine narrative ;

- examen clinique et évaluation de la douleur (Dolodog, Dolocat, CSOM : Client-Specific Outcome Measures, tracker d'activité) ;

- diagnostic de la douleur et de ses mécanismes physiopathologiques ;

- traitement multimodal et pluridisciplinaire de la douleur ;

- observance et éducation thérapeutique.
Le réseau CAPdouleur propose déjà des outils pour mettre en place cette consultation douleur : charte, leaflets, moniteur de consultation, procédures vidéo, etc.

\section{Projet de création d'UVETD}

Les UVETD sont des unités vétérinaires d'évaluation et de traitement de la douleur dédiées à la prise en charge plurimodale et pluridisciplinaire de la douleur chronique chez les animaux de compagnie.

La douleur chronique est définie comme une douleur persistante ou récurrente, induisant une détérioration fonctionnelle et des perturbations émotionnelles venant altérer significativement le comportement et la qualité de vie de l'animal.

Les UVETD assument la prise en charge des animaux douloureux chroniques avec une approche pluridisciplinaire, un projet thérapeutique individualisé et en tissant un lien indéfectible entre les spécialistes et les généralistes qui restent les seuls à adopter une approche globale de l'animal, au plus près du propriétaire.

Les UVETD deviennent des pôles de formation de proximité, proposant des parcours de formation, de la veille scientifique et des nouvelles méthodes d'apprentissage.

Les UVETD évoluent rapidement en centre de recherche clinique observationnelle pour améliorer les connaissances des douleurs neuropathiques et nociplastiques.

Les UVETD créent une plateforme d'échanges avec les CETD de médecine humaine pour faciliter la recherche clinique translationnelle au bénéfice du patient humain et du patient animal.

Un cahier des charges exigeant prévaut à ce type d'unité (projet d'équipe, formation continue des vétérinaires et des ASV, audit...). Une dizaine de structures devraient voir le jour à l'horizon 2022, portées par le réseau CAPdouleur.

\section{Lien douleur et bien-être de l'animal de compagnie}

En 2019 a été créé le Think Tank CAPwelfare qui est un groupe de réflexion pluridisciplinaire, réuni autour de la thématique du bien-être des animaux de compagnie. Convaincus que cette question méritait d'être explorée plus avant, ses membres ont conjugué leurs expertises pour offrir un panorama non exhaustif de ce que certains travaux scientifiques récents, mais également la pratique vétérinaire, peuvent nous apprendre sur le bien-être des animaux de compagnie. Un livre blanc (téléchargeable à l'adresse https://www.capdouleur.fr/livreblanc-capwelfare) est paru en juin 2019 pour nourrir le débat public sur cet enjeu de société, mais également fournir aux vétérinaires des clés de compréhension et 
des occasions de réflexions. En évitant de formuler des recommandations, le livre blanc invite les vétérinaires à se saisir de la thématique et à faire valoir leur expertise à partir de leur propre pratique.

Le livre blanc souhaite démontrer les nombreux parallèles et les points de convergence qui unissent douleur et BEA et la nécessité de mieux comprendre les interactions avec la cognition et les émotions.

Le modèle constructionniste a été théorisé récemment : la douleur se construit en fonction d'un contexte préexistant, façonné par des expériences singulières et un vécu douloureux (les traces mnésiques). La douleur s'échafaude en fonction d'un contexte environnemental révélateur d'une situation donnée à un instant $t$, modulé par les émotions et que les attentes perturbent (le catastrophisme chez l'humain, le pessimisme ou la résignation chez l'animal) ou minorent (l'optimisme). Dans ce modèle constructionniste, la douleur devient maladie avec des répercussions fonctionnelles, émotionnelles et comportementales, dégradant la qualité de vie et conduisant à un mal-être animal.

L'ANSES a proposé en 2018 : «Le bien-être d'un animal est l'état mental et physique positif lié à la satisfaction de ses besoins physiologiques et comportementaux, ainsi que de ses attentes. Cet état varie en fonction de la perception de la situation par l'animal ».

La théorie constructionniste de la douleur et la dernière définition du BEA tendent à se rejoindre, car les progrès des neurosciences et de l'éthologie bouleversent une approche moralisante condamnant les aspects négatifs (le mal-être) régis par des manques de libertés pour une vision éthique positive recherchant le bien-être sensoriel, émotionnel et mental des animaux. Ces définitions évolutives ont le grand intérêt d'élargir les champs thérapeutiques et de dépasser les strictes recommandations uniformes pour s'intéresser à l'histoire de l'être vivant douloureux et de sa vulnérabilité particulière.

Les animaux sont des êtres sensibles : ils ont assurément les supports neuronaux pour sentir des agressions thermiques, mécaniques ou chimiques, pour capter ce qui nuit, la nociception (du latin captere nocere). La sensibilité sensorielle évolue vers une sensibilité émotionnelle chez les animaux au système limbique développé et reprise par les Anglo-Saxons sous le terme d'animaux sentients (du latin sentiens, ressentant) : dorénavant, l'animal est décrit comme un être sensible doué d'émotions et doté de formes de conscience. Le Larousse reconnaît enfin dans son édition 2020 la sentience définit par « la capacité pour un être vivant à ressentir les émotions, la douleur, le bien-être, etc. et à percevoir de façon subjective son environnement et ses expériences de vie $»$.

Désormais, la douleur devient aussi chez les animaux une perception fluctuante émotionnelle et cognitive d'une sensation nociceptive : la douleur est construite par rapport à un contexte préexistant d'un vécu douloureux et en fonction d'une situation environnementale : la situation, les émotions, les attentes de l'animal, le lien avec le propriétaire ont une influence, positive comme négative.

Les progrès des neurosciences et de la neuro-imagerie ont conforté l'évidence clinique du lien entre douleurs chroniques, émotions et cognition, qui partagent les mêmes neurotransmetteurs, et des voies de communication et des structures cérébrales communes [22]. Ces rapports sont complexes, interactifs et très souvent autoaggravants.

Les émotions négatives et positives majorent ou minorent la perception de la douleur des animaux. Le déclin cognitif est similaire chez l'humain et le chien : il porte sur l'apprentissage, la mémoire, l'attention et les fonctions exécutives. Chez l'animal de laboratoire, des déficits cognitifs apparaissent en cas de douleur expérimentale chronique. Il a été suggéré que la douleur chronique pouvait provoquer des difficultés de contrôle attentionnel, notamment parce que les mêmes régions cérébrales sont impliquées prioritairement dans le traitement attentionnel et l'expérience de la douleur [23]. Enfin, la douleur chronique peut engendrer des changements structuraux, dont des réductions de la matière grise au niveau du cortex dorsolatéral préfrontal et du thalamus [24].

La douleur entraîne donc des conséquences cognitives et, inversement, les modifications de la cognition peuvent moduler la douleur. Le stress engendré par la douleur est d'ailleurs au centre du processus d'altération cognitive essentiellement par dysfonctionnement du système hypothalamohypophysaire source de remaniements de la région de l'hippocampe [25].

La prise en charge raisonnée et protectrice des douleurs chroniques doit dorénavant s'intéresser à ce triptyque « douleur-émotion-cognition » en explorant davantage l'expérience construite de la douleur.

\section{Conclusion}

Les actualités autour de la douleur animale sont particulièrement riches, et la profession vétérinaire montre un fort intérêt à une prise en charge moderne de la douleur : formation continue, e-santé, réseau de partage de connaissances, consultation douleur, UVETD, lien douleur et BEA... La vitalité de cet engagement se traduit par une amélioration des pratiques analgésiques dans les établissements de soins vétérinaires et le regroupement en réseau pour un partage des connaissances. Le projet CAPdouleur a reçu le prix de l'ordre des vétérinaires en 2015 pour récompenser, selon le président Michel Baussier : "Une démarche à la fois scientifique, éthique, managériale et de communication ». Selon le chercheur et professeur de médecine Guy Simonnet, le réseau CAPdouleur est un «Pari de modernité dans lequel les praticiens pourront s'approprier un patrimoine 
vétérinaire, bâti sur le partage des expériences individuelles et collectives ».

Selon Bernard Neveux de Vétitude (www.vetitude.fr), l'initiative CAPdouleur, unique à l'international, répond à tous les critères d'une stratégie de rupture apportée par les nouvelles technologies car :

- elle modifie le management de l'activité quotidienne des vétérinaires ;

- elle propose aux praticiens d'utiliser les données pour corriger leurs pratiques et pour se former : ils n'ont plus qu'à aller au bout de la démarche en l'insérant dans la première blockchain vétérinaire digne de ce nom au plan national ;

- elle inclut les premiers éléments d'intelligence artificielle ;

- elle offre ce que tout utilisateur de nouvelles technologies recherche : la liberté et l'autonomie vis-à-vis des anciens modèles scientifiques, de formation et de communication vétérinaires.

Le programme de prise en charge de la douleur instruit par le projet CAPdouleur est aujourd'hui le plus abouti dans le contexte Vetfuturs initié en France conjointement par le Syndicat national des vétérinaires d'exercice libéral et par le Conseil national de l'ordre des vétérinaires : Vetfuturs prépare l'avenir de la profession vétérinaire pour les 15 ans à venir, à la lumière des changements humains, sociaux, économiques, techniques et scientifiques impactant déjà l'exercice quotidien des vétérinaires.

Liens d'intérêts : l'auteur déclare avoir une activité de formation et de conseil en analgésie ; il est fondateur du réseau CAPdouleur.

\section{Références}

1. Rodkey EN, Pillai Riddell R (2013) The infancy of infant pain research: the experimental origins of infant pain denial. J Pain $14: 338-50$

2. Anand KJ, Hickey PR (1987) Pain and its effects in the human neonate and fetus. $\mathrm{N}$ Engl $\mathrm{J}$ Med 317:1321-9. doi: 10.1056/NEJM198711193172105

3. Taddio A, Gurguis M, Koren G (2002) Lidocaine-prilocaine cream versus tetracaine gel for procedural pain in children. Ann Pharmacother 36:687-92

4. Knapp DW, Henry CJ, Widmer WR, et al (2013) Randomized trial of cisplatin versus firocoxib versus cisplatin/firocoxib in dogs with transitional cell carcinoma of the urinary bladder. $\mathrm{J}$ Vet Intern Med 27:126-33

5. Arenas C, Peña L, Granados-Soler JL, Pérez-Alenza MD (2016) Adjuvant therapy for highly malignant canine mammary tumours: COX-2 inhibitor versus chemotherapy: a case-control prospective study. Vet Rec 179

6. Rausch-Derra L, Huebner M, Wofford J, Rhodes L (2016) A prospective, randomized, masked, placebo-controlled multisite clinical study of grapiprant, an EP4 prostaglandin receptory anta- gonist (PRA), in dogs with osteoarthritis. J Vet Intern Med 30:756-63

7. Budsberg Steven C, Torres Bryan T, Kleine Stephanie A, et al (2018) Lack of effectiveness of tramadol hydrochloride for the treatment of pain and joint dysfunction in dogs with chronic osteoarthritis. JAVMA 252: 427-432

8. Moore S (2016) Managing neuropathic pain in dogs. Front Vet Sci 3:12 (Published online 2016 Feb 22)

9. Nugent SM, Morasco BJ, O'Neil ME, et al (2017) The effects of cannabis among adults with chronic pain and an overview of general harms: a systematic review. Ann Intern Med 167:319-31

10. Fahie Maria A (2013) A randomized controlled trial of the efficacy of autologous platelet therapy for the treatment of osteoarthritis in dogs. JAVMA 243:1291-7

11. Taroni M, Cabon Q, Fèbre M, et al (2017) Evaluation of the effect of a single intra-articular injection of allogeneic neonatal mesenchymal stromal cells compared to oral non-steroidal antiinflammatory treatment on the postoperative musculoskeletal status and gait of dogs over a 6-month period after tibial plateau leveling osteotomy: a pilot study. Front Vet Sci 4 (article 83) :1-11

12. Cabon Q, Febre M, Gomez N, et al (2019) Long-term safety and efficacy of single or repeated intra-articular injection of allogeneic neonatal mesenchymal stromal cells for managing pain and lameness in moderate to severe canine osteoarthritis without antiinflammatory pharmacological support: pilot clinical study. Front Vet Sci (in press)

13. Lascelles BD (2015) A canine-specific anti-nerve growth factor antibody alleviates pain and improves mobility and function in dogs with degenerative joint disease-associated pain. BMC Vet Res 11:101

14. Gruen ME, Thomson AE, Griffith EH, et al (2016) A felinespecific anti-nerve growth factor antibody improves mobility in cats with degenerative joint disease-associated pain: a pilot proof of concept study. J Vet Intern Med 30:1138-48

15. Brown DC, Boston RC, Farrar JT (2013) Comparison of force plate gait analysis and owner assessment of pain using the canine brief pain inventory in dogs with osteoarthritis. J Vet Intern Med 27:22-30

16. Hielm-Björkman AK, Rita H, Tulamo RM (2009) Psychometric testing of the Helsinki Chronic Pain Index by completion of a questionnaire in Finnish by owners of dogs with chronic signs of pain caused by osteoarthritis. Am J Vet Res 70:727-34

17. Walton MB, Cowderoy E, Lascelles D, et al (2013) Evaluation of construct and criterion validity for the "Liverpool osteoarthritis in dogs" (Load) clinical metrology instrument and comparison to two other instruments. PLoS One 8:1-10

18. Brown DC, Boston RC (2010) Use of an activity monitor to detect response to treatment in dogs with osteoarthritis. J Am Vet Med Assoc 37:66-70

19. Wernham BGJ, Trumpatori B, Hash J, et al (2011) Dose reduction of meloxicam in dogs with osteoarthritis associated pain and impaired mobility. J Vet Intern Med 25:1298-305

20. Dahl JB, Moiniche S (2004) Pre-emptive analgesia. Br Med Bull 71:13-27

21. Bromley L (2006) Pre-emptive analgesia and protective premedication. What is the difference? Biomed Pharmacother 60:336-40

22. Laurent B (2015) Douleur chronique : son retentissement émotionnel et cognitif. Bull Acad Natl Med 199:543-54 (séance du 5 mai 2015)

23. Wade JB, Hart RP (2002) Attention and the stages of pain processing. Pain Med 3:30-8

24. Apkarian AV, Sosa Y, Sonty S, et al (2004) Chronic back pain is associated with decreased prefrontal and thalamic gray matter density. J Neurosci 24:10410-5

25. Mc Ewen BS (1999) Stress and the aging hippocampus. Front Neuroendocrinol 20:49-70 